\title{
MFS multidrug transporters in pathogenic fungi: do they have real clinical impact?
}

\section{Catarina Costa, Paulo J. Dias, Isabel Sá-Correia and Miguel C. Teixeira*}

Biological Sciences Research Group, Department of Bioengineering, Instituto Superior Técnico, IBB - Institute for Biotechnology and Bioengineering, Universidade de Lisboa, Lisbon, Portugal

\section{Edited by:}

Paula Duque, Instituto Gulbenkian de Ciência, Portugal

Reviewed by:

Dominique Sanglard, University of Lausanne and University Hospital Center, Switzerland

Frederic Devaux, Universite Pierre et Marie Curie, France

${ }^{*}$ Correspondence:

Miguel C. Teixeira, Biological

Sciences Research Group, Centro de Engenharia Biológica e Química, Instituto Superior Técnico, IBB Institute for Biotechnology and

Bioengineering, Universidade de Lisboa, Av. Rovisco Pais,

1049-001 Lisbon, Portugal

e-mail:mnpct@ist.utl.pt

Infections caused by opportunistic fungal pathogens have reached concerning numbers due to the increase of the immunocrompromised human population and to the development of antifungal resistance. This resistance is often attributed to the action of multidrug efflux pumps, belonging to the ATP-binding cassette $(A B C)$ superfamily and the major facilitator superfamily (MFS). Although many studies have focused on the role of $A B C$ multidrug efflux transporters, little is still known on the part played by the Drug: $\mathrm{H}^{+}$ Antiporter (DHA) family of the MFS in this context. This review summarizes current knowledge on the role in antifungal drug resistance, mode of action and phylogenetic relations of DHA transporters, from the model yeast $S$. cerevisiae to pathogenic yeasts and filamentous fungi. Through the compilation of the predicted DHA transporters in the medically relevant Candida albicans, C. glabrata, C. parapsilosis, C. Iusitaniae, C. tropicalis, C. guilliermondii, Cryptococcus neoformans, and Aspergillus fumigatus species, the fact that only $5 \%$ of the DHA transporters from these organisms have been characterized so far is evidenced. The role of these transporters in antifungal drug resistance and in pathogen-host interaction is described and their clinical relevance discussed. Given the knowledge gathered for these few DHA transporters, the need to carry out a systematic characterization of the DHA multidrug efflux pumps in fungal pathogens, with emphasis on their clinical relevance, is highlighted.

Keywords: multidrug resistance efflux pumps, drug:H+ antiporters, antifungal drug resistance, pathogenic fungi, Candida species

\section{INTRODUCTION}

The multidrug resistance (MDR) phenomenon, characterized by the simultaneous acquisition of resistance to chemically and structurally different compounds (Sá-Correia et al., 2009; Morschhauser, 2010), poses a severe problem in the treatment of fungal infections. This is particularly relevant since the number of infections caused by opportunistic fungal pathogens has increased considerably in recent years due to the widespread use of antifungal drugs in immunocompromised patients, such as individuals undergoing chemotherapy, HIV-infected, or AIDS patients (Morschhauser, 2010).

There are mainly four mechanisms by which a cell can deal with a toxic compound: (i) drug target alteration, (ii) drug inactivation, (iii) reduced uptake, or (iv) active extrusion (Ernst et al., 2010). The latter occurs mainly due to the action of membrane transporters which belong to one of two superfamilies in fungi: the ATP-binding cassette superfamily $(\mathrm{ABC})$ and the major facilitator superfamily (MFS) (Cannon et al., 2009; Sá-Correia et al., 2009; Morschhauser, 2010). The role of the ABC multidrug transporters in antifungal resistance in clinical isolates has been well characterized in the past decades. However, much less attention has been drawn to the expected role of the Drug: $\mathrm{H}^{+}$Antiporter (DHA) family of the MFS.

In this paper, knowledge gathered so far on the role of the DHA family in antifungal drug resistance is reviewed, with emphasis on its clinical relevance. Although starting from what was found, in this context, in the model yeast Saccharomyces cerevisiae, particular focus is given to the DHA transporters found to occur, based on phylogenetic analysis, in pathogenic yeasts of the Candida genus and also in Cryptococcus neoformans, and Aspergillus fumigatus. Current challenges and expected impact of research in this topic is finally discussed.

\section{THE DRUG:H+ ANTIPORTER FAMILY: LESSONS FROM SACCHAROMYCES CEREVISIAE}

Upon the release of the complete Saccharomyces cerevisiae genome sequence (Goffeau et al., 1996), a total of 22 transporters belonging to the MFS were identified and clustered into two families: the drug: $\mathrm{H}^{+}$antiporter family 1 (DHA1) and 2 (DHA2). These families differ mainly in the number of transmembrane spans (TMS), with the first having 12 and the second 14 TMS (Sá-Correia et al., 2009).

S. cerevisiae has 12 DHA1 and 10 DHA2 transporters, most of which have been implicated in MDR, while some are yet to be characterized (reviewed in Sá-Correia et al., 2009). Most of these transporters were found to confer resistance to a large number of unrelated chemicals. Given this apparent promiscuity, their exact mode of action as multidrug resistance determinants is controversial. For most S. cerevisiae DHA transporters a role in the physiology of the cell was further identified. That is the case of ScTpo1-4 and ScQdr3, which confer resistance to toxic levels of polyamines (Tomitori et al., 2001; Albertsen et al., 2003; 
Teixeira et al., 2011), of ScDtr1, that facilitates the translocation of bisformyl dityrosine through the prospore membrane during spore wall maturation (Felder et al., 2002), of ScQdr2, involved in potassium homeostasis (Vargas et al., 2007), and of ScAqr1, that has been proposed to excrete amino acids, such as homoserine and threonine (Velasco et al., 2004).

Most of the characterized transporters of S. cerevisiae, both from DHA1 and DHA2 subfamilies, confer resistance to more than one different growth inhibitory compound, with ScTpol being, by far, the one with the broadest range of predicted substrates (Sá-Correia et al., 2009). Among these compounds, some are of particular relevance in the combat against fungal phytopathogens. For example, ScFlr1 was found to confer resistance against the agricultural fungicides benomyl (Brôco et al., 1999) and mancozeb (Teixeira et al., 2008, 2010). The most interesting finding, however, is that, even though this is a non-pathogenic species, a lot of these efflux pumps confer resistance to or are upregulated by widely used clinically relevant antifungal drugs, such as fluconazole (ScFlr1, ScQdr1, ScYhk8, and ScAzr1) (Tenreiro et al., 2000; Nunes et al., 2001; Barker et al., 2003), ketoconazole (ScAqr1, ScQdr1, ScQdr2, ScAzr1) (Tenreiro et al., 2000, 2002; Nunes et al., 2001), itraconazole (ScYhk8) (Barker et al., 2003) and caspofungin (ScTpo1) (Markovich et al., 2004). Some of them even confer resistance to more than one of these antifungal agents, as is the case of ScAzr1 and ScQdr1.

The paradigmatic case of the study of DHA transporters in $S$. cerevisiae highlights three important facts: (1) the existence and, thus, function of 20 out of 22 of these transporters remained concealed until the genome sequence was disclosed; (2) these transporters were indeed found to confer multidrug resistance, but also to play important roles in S. cerevisiae physiology; and (3) there is significant functional overlap between these transporters, making the discovery of their function a bigger challenge. The functional analysis of the $S$. cerevisiae DHA transporters provides clues on the function of homolog transporters from pathogenic yeast and filamentous fungi, but also suggests that their role tends to be elusive.

\section{DRUG: ${ }^{+}$ANTIPORTER FAMILY: DISSEMINATION IN PATHOGENIC YEAST AND FUNGI}

The Candida DHA1 and DHA2 transporters considered in this review are those predicted to be encoded in the genomes of $C$. albicans, C. tropicalis, C. parapsilosis, C. guilliermondii, C. lusitaniae, and C. glabrata (Dias et al., 2010; Dias and Sá-Correia, 2013 , in press). The DHA1 and DHA2 proteins encoded in the genomes of $A$. fumigatus and $C$. neoformans were identified using the annotation provided by the Aspergillus Genome Database (AspGD_http://www.aspgd.org/) and, based on the BlastP algorithm, using the $S$. cerevisiae DHA1 and DHA2 proteins to query the Genbank database, respectively. A total of 185 full-size DHA1 proteins and 85 full-size DHA2 proteins were found to be encoded in these eight fungal species, and clustered according to the phylogenetic trees displayed in Figure 1.

This review considers DHA1 proteins belonging to 20 previously reported phylogenetic clusters, labeled A to V (Dias et al., 2010; Dias and Sá-Correia, in press), and describes the occurrence of 8 new clusters, which include only A. fumigatus genes (clusters
1-4 and 6-7) or A. fumigatus and C. neoformans genes (cluster 5) (Figure 1). It becomes clear that the A. fumigatus genome encodes a higher number and higher diversity of DHA1 proteins compared with hemiascomycetous genomes. However, A. fumigatus lacks homologs to the S. cerevisiae Dtr1, Hol1, Tpo4 and Flr1 transporters (Figure 1). The genome of the basidiomycete yeast $C$. neoformans comprises a total of 9 DHA1 proteins, including homologs of S. cerevisiae Qdr1/Qdr2/Aqr1, Tpo2/Tpo3 and Flr1 proteins, one homolog of the C. albicans Tmp1/Tmp2 proteins and three proteins residing in the new phylogenetic cluster 5 (Figure 1).

The DHA2 proteins considered or identified in this study are distributed throughout 8 previously reported phylogenetic clusters (Dias and Sá-Correia, 2013), plus 11 newly defined clusters, composed only by A. fumigatus and/or C. neoformans proteins (labeled 1-11). The A. fumigatus genome encodes a total of 33 DHA2 transporters, two thirds of which reside in clusters lacking hemiascomycetous DHA2 members. A. fumigatus genome does not include homologs of Sge1/Azr1/Vba3/Vba5 or Atr1/YMR279C proteins. A total of 7 DHA2 proteins were found in the C. neoformans genome, among which only one S. cerevisiae homolog is found, clustering with Vba1/Vba2 (Figure 1).

Altogether, the abundance of predicted DHA transporters in the Candida, Cryptococcus and Aspergillus species considered, reaching 87 for A. fumigatus, is very high, strongly suggesting that these transporters must play an important function in these organisms. Among almost 300 ORFs compiled in Figure 1 only 12 have already been characterized, corresponding to around 5\% of the total number. Their predicted involvement in drug resistance highlights the importance of characterizing all of them in a systematic way.

\section{ROLE OF DHA TRANSPORTERS IN ANTIFUNGAL DRUG RESISTANCE}

Only a few DHA transporters have been linked to antifungal drug resistance in pathogenic fungi (Table 1). This appears to be mostly due to lack of characterization efforts. The C. albicans drug efflux pump Mdrl was the first protein identified as a multidrug MFS transporter in a pathogenic fungus. CaMdr1 expression was found to confer resistance to fluconazole and ketoconazole, but not to itraconazole or miconazole, in S. cerevisiae, while overexpression in C. albicans leads to fluconazole resistance (Goldway et al., 1995; Hiller et al., 2006). CaNag3 and CaNag4 have also been shown to confer resistance to several antifungal drugs, such as cycloheximide, 4-nitroquinoline- $N$-oxide and 1-10 phenanthroline, being thus proposed to be multidrug efflux pumps (Yamada-Okabe and Yamada-Okabe, 2002). The $C$. albicans DHA1 transporter CaFlu1 was found to complement fluconazole hypersusceptibility in a $S$. cerevisiae $\Delta p d r 5$ mutant, but not to have a significant role in fluconazole resistance in C. albicans (Calabrese et al., 2000). On the other hand, Flul was more recently shown to confer resistance to the salivary human antimicrobial peptide histatin 5, playing a direct role in its efflux from $C$. albicans cells, thus reducing histatin 5 toxicity (Li et al., 2013). Of a total of 26 DHA transporters found to be encoded by C. albicans genome, 18 are still uncharacterized. 
A

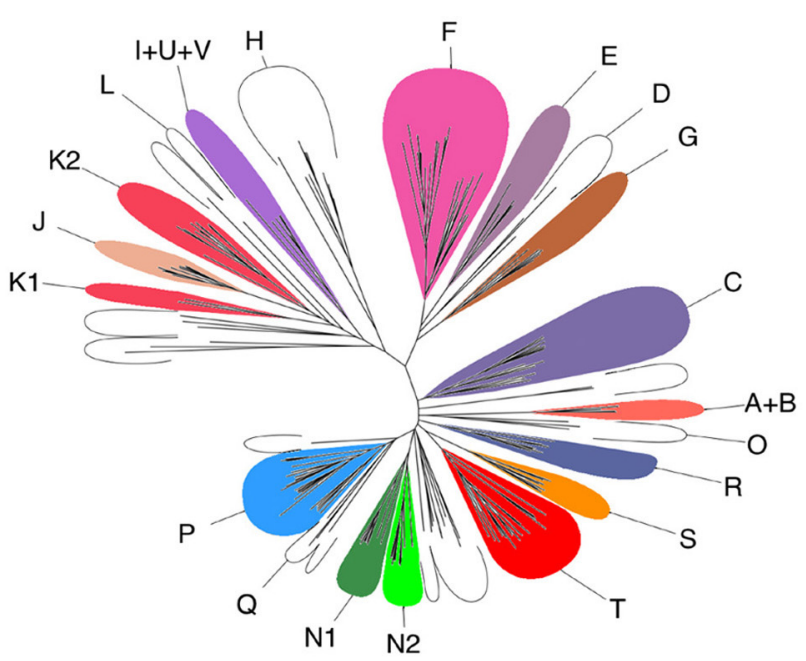

B

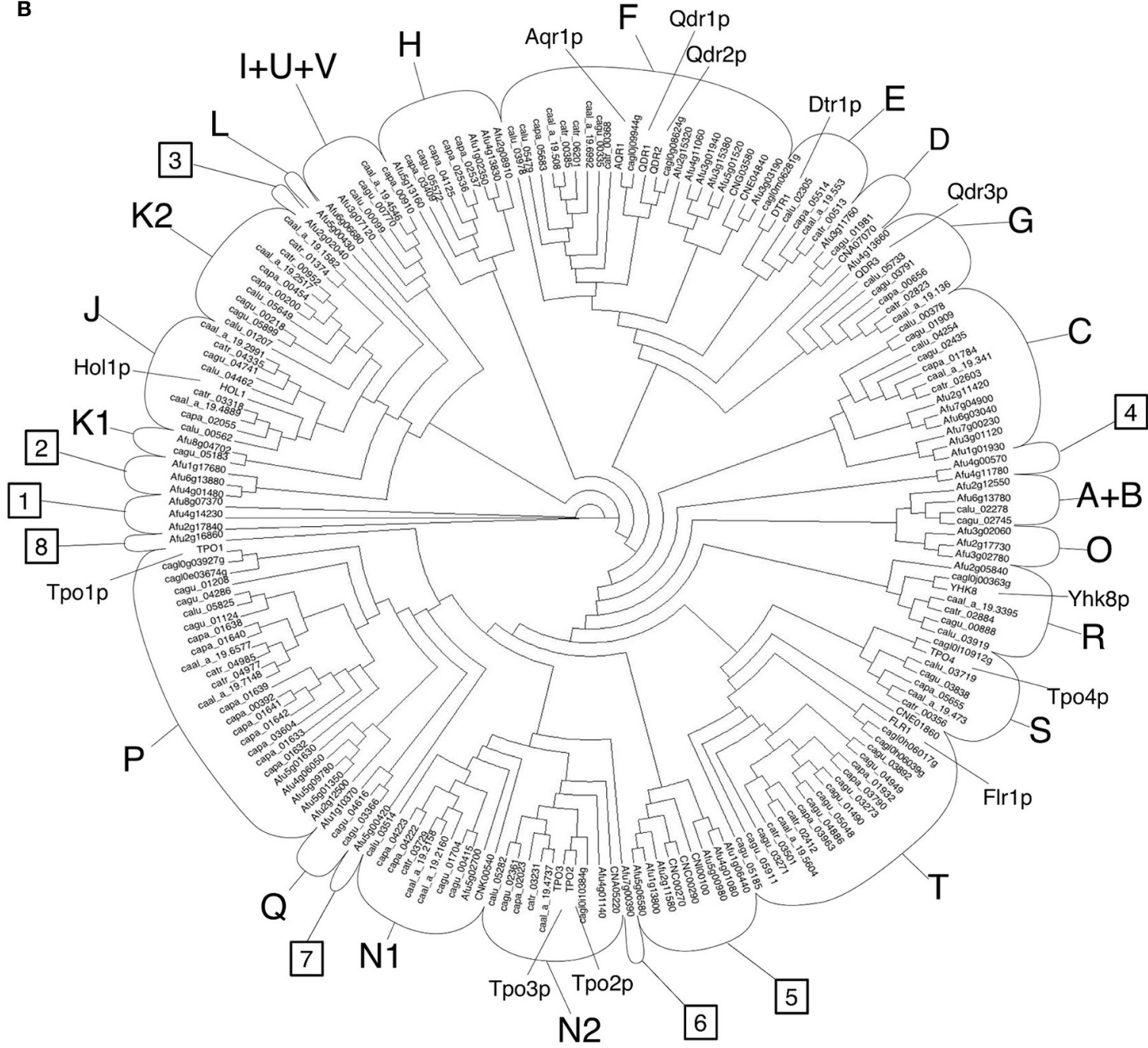

FIGURE 1 | Continued 
C

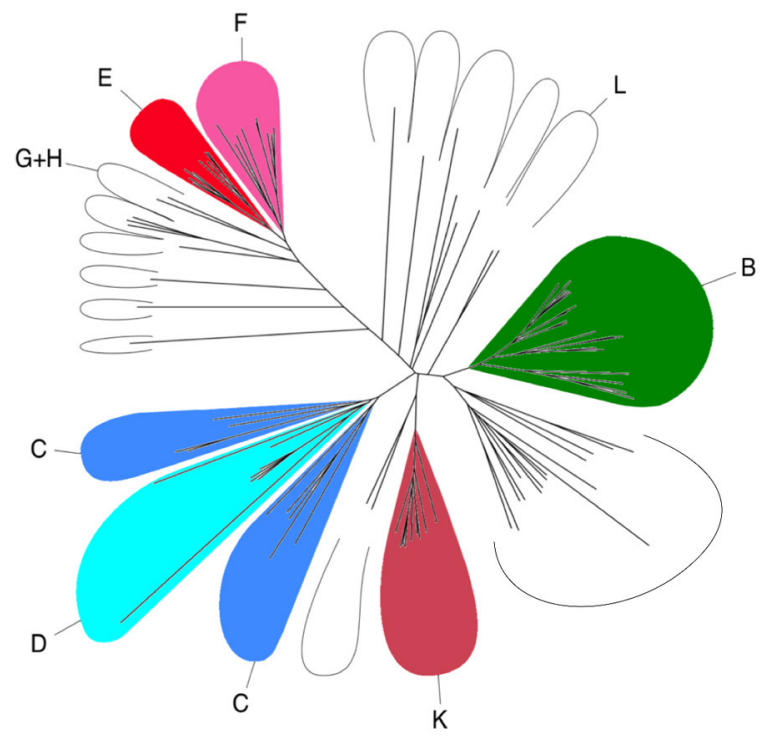

D

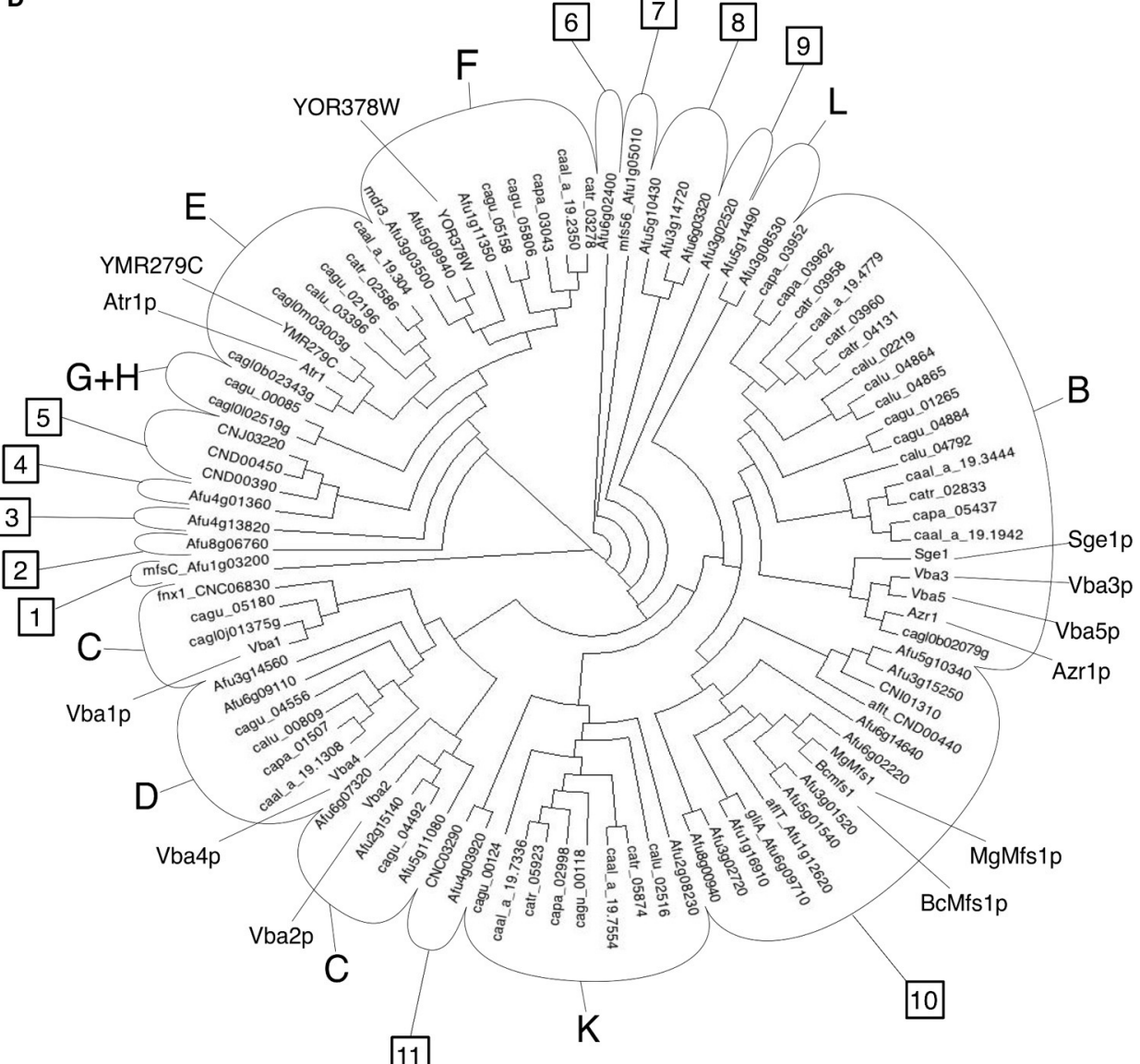

FIGURE 1 | Phylogenetic analysis of DHA1 and DHA2 transporters gathered from the $S$. cerevisiae $\mathrm{S} 288 \mathrm{C}$ reference strain and from eight clinically important fungal species - $C$. albicans, $C$. tropicalis, $C$. parapsilosis, $C$. guilliermondii, $C$. Iusitaniae, $C$. glabrata, $A$. fumigatus and $C$. neoformans - using the PROTDIST/NEIGHBOR packages of PHYLIP suite, as detailed in (Dias and Sá-Correia, 2013, in press). (A)
Radial phylogram showing the amino acid sequence similarity distances between these 197 full-size DHA1 transporters. (B) Circular cladogram showing the corresponding tree topology. The DHA1 proteins are distributed into 20 known phylogenetic clusters, labeled using letters and based on previous cluster annotation (Dias and Sá-Correia, in press), and 8 new

(Continued) 


\section{FIGURE 1 | Continued}

phylogenetic clusters (clusters 1-4 and 6-7) comprising only members of the filamentous fungi. (C) Radial phylogram showing the amino acid sequence similarity distances between these 95 full-size DHA2 transporters. (D) Circular cladogram showing the corresponding tree topology. The DHA2 proteins are distributed into 8 known phylogenetic clusters, labeled using letters and based on a previous cluster annotation
(Dias and Sá-Correia, 2013), and 11 new phylogenetic clusters (clusters 1-11) comprising only members of the filamentous fungi. The DHA1 and the DHA2 proteins encoded in the genome of $S$. cerevisiae S288c strain and biochemically characterized MgMfs1 and BcMfs1 DHA2 fungal proteins were used as functional reference and the corresponding names are indicated in the phylogenetic trees. The ARN and GEX proteins (Dias and Sá-Correia, 2013), were not included in this analysis.
Close homologs to CaMdr1 were found in both $C$. dubliniensis and $C$. tropicalis. In the first, inactivation of the gene was shown to result in an increased susceptibility to fluconazole (Wirsching et al., 2001), while in the latter its expression increased when cells were treated with increasing concentrations of fluconazole and, at the same time, developed cross-resistance to azoles and terbinafine (Barchiesi et al., 2000).

C. glabrata, a pathogenic yeast that is phylogenetically closer to $S$. cerevisiae than to other Candida species, shows a high level of intrinsic resistance to fluconazole. However, the DHA transporter CgFlr1, a CaMdr1 homolog, was found to confer resistance to benomyl, in a strain that was already a mutant for both CDR1 and CDR2 genes, but not to fluconazole or other azoles (Chen et al., 2007). More recently, three additional DHAl transporters were characterized in what concerns their contribution to the MDR phenomenon and their role in antifungal drug resistance. CgQdr2, CgAqr1, and CgTpo3 were found to be involved in multidrug resistance as they confer resistance to a wide variety of toxic compounds (Costa et al., 2013a,b, 2014), the most relevant being, for CgQdr2, the imidazoles clotrimazole, miconazole, tioconazole, and ketoconazole (Costa et al., 2013b), for CgAqr1, the antifungal drugs flucytosine and, less significantly, clotrimazole (Costa et al., 2013a) and for CgTpo3 the azole antifungals clotrimazole, ketoconazole, miconazole, tioconazole, itraconazole, and fluconazole (Costa et al., 2014). The role of CgAqr1 and CgTpo3 transporters in acetic acid (Costa et al., 2013a) and polyamine (Costa et al., 2014) resistance, respectively, is also an interesting feature given that Candida species have often to thrive in acetic acid and polyamine rich environments such as the vaginal mucosa or the urogenital tract, respectively.

For Aspergillus fumigatus, the transporter Mfs56 from the DHA2 family has been characterized and found to have a role in the resistance to itraconazole, posaconazole and ravuconazole (Bowyer et al., 2012). Also, the AfMdr3 transporter has been found to be overexpressed in cells treated with amphotericin B (Gautam et al., 2008). Interestingly, two characterized multidrug transporters from the phytopathogenic fungi Botrytis cinerea, Bcmfs1 (Hayashi et al., 2002), and Mycosphaerella graminicola, Mgmfs1 (Roohparvar et al., 2007), are shown to cluster together with cluster 10 (Figure 1). Mgmfs1 in particular was found to confer resistance to azoles and cycloheximide, among many other tested compounds (Roohparvar et al., 2007), suggesting that a similar function may be performed by the remaining members of the DHA2 cluster 10 .

\section{DRUG:H+ ANTIPORTER CLINICAL IMPACT: WHAT'S KNOWN AND WHAT IS THERE TO BE FOUND}

The clinical impact of the DHA transporters in fungal pathogens is yet to be fully investigated. So far, only a couple of them have been shown to effectively contribute to the development, progression, or persistence of the infection in the host (Table 1). That is the case of $C$. albicans Mdr1, which has been found to be consistently expressed in high values in fluconazole resistant clinical isolates (Wirsching et al., 2000a,b), the same being observed for C. dubliniensis Mdr1 (Wirsching et al., 2001). CaNag3 and CaNag4 have also been shown to be involved in C. albicans virulence, as adult mice infected intravenously with $10^{6}$ or $10^{7}$ cells of the $\Delta$ canag3, $\Delta$ canag 4 or $\Delta$ canag 3 canag4 mutants lived for at least 4 weeks (in the lowest cell concentration for single mutants, and both concentrations for double mutant), compared with a maximum of 11 days for the lowest concentration of the wild type (Yamada-Okabe and Yamada-Okabe, 2002). Very recently, the deletion of C. albicans Qdr1, Qdr2, and Qdr3 transporters was found to lead to defects in biofilm architecture and thickness and to attenuate virulence in a mouse model (Shah et al., 2014). Although the exact mechanism underlying this observation was not clarified, the expression of these transporters was found to have a deep effect in membrane lipid composition, which may underly the observed phenotypes (Shah et al., 2014). The C. glabrata DHA transporters CgAqr1 and CgTpo3 were also suggested to contribute to the survival of this pathogen within the host, as they confer resistance to acetic acid (Costa et al., 2013a) and polyamines (Costa et al., 2014), respectively, which accumulate to inhibitory concentrations in Candida infection prone environments. Indeed, the concentration of lactic or acetic acid can reach up to $125 \mathrm{mM}$ in the vaginal tract, particularly under bacterial vaginosis (Chaudry et al., 2004), whereas polyamine concentrations can reach up to $15 \mathrm{mM}$ spermine, $5 \mathrm{mM}$ spermidine, and $3 \mathrm{mM}$ putrescine in the urogenital tract (Tyms, 1989).

Of the high number of MFS transporters that fungal genomes encode, only a reduced amount has been shown to be involved in MDR or to be able to confer resistance to antifungal drugs, and an even lower number has been described as clinically relevant, so far. The lack of information on the clinical impact of fungal DHA transporters may be associated with the types of experiments carried out in this context. For instance, in C. albicans, the upregulation of the ABC transporters CaCDR1 and $C a C D R 2$ in fluconazole-resistant isolates, which has used to assess their clinical relevance, was assessed mostly by targeted gene expression analysis (Sanglard et al., 1995, 1997), or in a few cases by the genome-wide analysis of the transcriptional response to antifungal drugs (Karababa et al., 2004; Liu et al., 2007). Furthermore, the impact of these transporters may not depend merely on their over-expression. For example, polymorphic mutant alleles of CaMDR1 have been found among clinical isolates, inducing distinct drug resistance profiles (Gupta et al., 1998). More detailed analysis of the expression and sequence variation of DHA encoding genes in clinical isolates is likely to 
Table 1 | DHA1 and DHA2 transporters predicted from the genome sequences of Candida spp., Aspergillus fumigatus, and Cryptococcus neoformans.

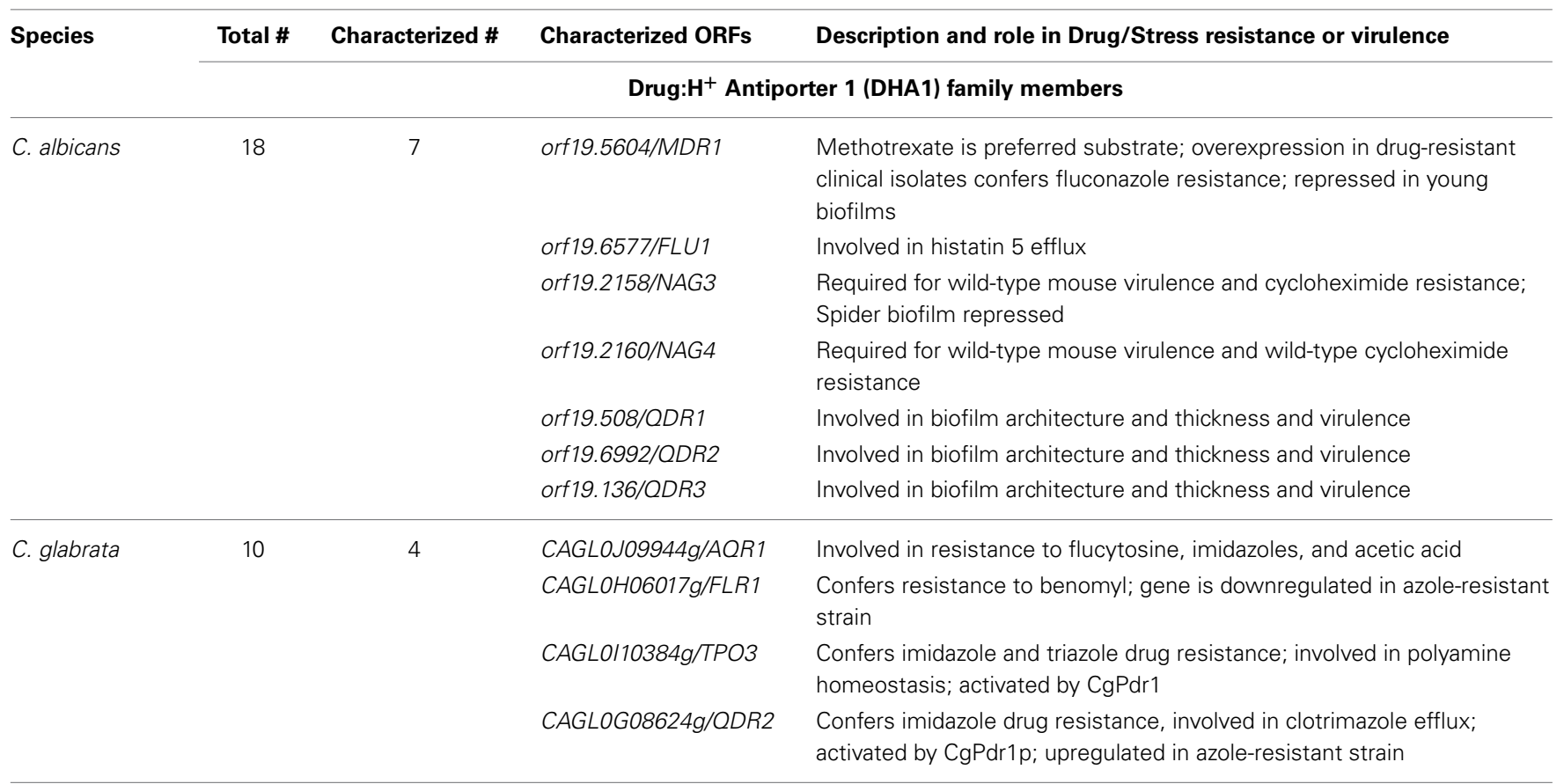

\begin{tabular}{lcccc}
\hline C. parapsilosis & 28 & 0 & & \\
C. lusitaniae & 17 & 0 & & \\
C. tropicalis & 18 & 0 & & \\
C. guilliermondii & 31 & 0 & CNA07070 & Dityrosine transporter \\
C. neoformans & 9 & 1 & & \\
A. fumigatus & 54 & 0 & &
\end{tabular}

\begin{tabular}{lccll}
\hline & & & \multicolumn{1}{c}{ Drug: $\mathbf{H}^{+}$Antiporter 2 (DHA2) family members } \\
\hline C. albicans & 8 & 1 & orf19.2350 & Affects filamentous growth \\
C. glabrata & 5 & 0 & & \\
C. parapsilosis & 6 & 0 & & \\
C. lusitaniae & 7 & 0 & & \\
C. tropicalis & 8 & 0 & & \\
C. guilliermondii & 11 & 0 & CNC03290 & Tetracycline efflux protein \\
C. neoformans & 7 & 2 & Afu6g09710/gliA & Predicted glioxin transporter \\
& 33 & 2 & Afu1g05010/mfs56 & Mutation causes increased azole sensitivity
\end{tabular}

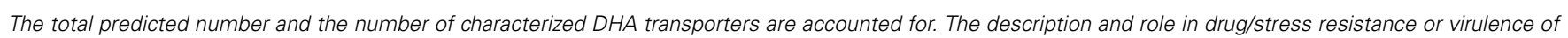
the few characterized DHA is highlighted (adapted from CGD and AspGD).

provide clues on their impact in the acquisition of MDR in clinical settings.

\section{CONCLUSIONS AND PERSPECTIVES}

Given their predicted role in antifungal drug resistance, a few DHA transporters in pathogenic yeasts and fungi have already been characterized. So far, their characterization suggested interesting roles in antifungal drug resistance and, in some cases, in host-pathogen interactions. However, only $5 \%$ of the predicted DHA transporters in the 8 fungal pathogens considered in this review have ever been studied. It appears, thus, crucial to study this underappreciated family of transporters, as they may provide decisive insights into the mechanisms underlying antifungal drug resistance that may guide more efficient fungal infection diagnosis, prophylaxis, and therapeutics. Furthermore, unlike what has been observed for $\mathrm{ABC}$ drug efflux pumps, which are widespread from bacteria to man, the DHA family appears to be strictly conserved within bacteria and fungi, turning these proteins into interesting candidates as targets for the development of new antifungal drugs. 


\section{ACKNOWLEDGMENTS}

Research conducted by the authors within the scope of this paper was supported by Fundação para a Ciência e a Tecnologia (FCT; contract PTDC/EBB-BIO/119356/2010; PhD and post-doc scholarships to Catarina Costa and Paulo J. Dias, contracts SFRH/BD/69809/2010 and SFRH/BPD/74618/2010, respectively).

\section{REFERENCES}

Albertsen, M., Bellahn, I., Kramer, R., and Waffenschmidt, S. (2003). Localization and function of the yeast multidrug transporter Tpolp. J. Biol. Chem. 278, 12820-12825. doi: 10.1074/jbc.M210715200

Barchiesi, F., Calabrese, D., Sanglard, D., Falconi Di Francesco, L., Caselli, F., Giannini, D., et al. (2000). Experimental induction of fluconazole resistance in Candida tropicalis ATCC 750. Antimicrob. Agents Chemother. 44, 1578-1584. doi: 10.1128/AAC.44.6.1578-1584.2000

Barker, K. S., Pearson, M. M., and Rogers, P. D. (2003). Identification of genes differentially expressed in association with reduced azole susceptibility in Saccharomyces cerevisiae. J. Antimicrob. Chemother. 51, 1131-1140. doi: $10.1093 / \mathrm{jac} / \mathrm{dkg} 217$

Bowyer, P., Mosquera, J., Anderson, M., Birch, M., Bromley, M., and Denning, D. W. (2012). Identification of novel genes conferring altered azole susceptibility in Aspergillus fumigatus. FEMS Microbiol. Lett. 332, 10-19. doi: 10.1111/j.15746968.2012.02575.x

Brôco, N., Tenreiro, S., Viegas, C. A., and Sá-Correia, I. (1999). FLR1 gene (ORF YBR008c) is required for benomyl and methotrexate resistance in Saccharomyces cerevisiae and its benomyl-induced expression is dependent on Pdr3 transcriptional regulator. Yeast 15, 1595-1608. doi: 10.1002/(SICI)10970061(199911)15:15\&lt;1595::AID-YEA484\&gt;3.0.CO;2-6

Calabrese, D., Bille, J., and Sanglard, D. (2000). A novel multidrug efflux transporter gene of the major facilitator superfamily from Candida albicans (FLU1) conferring resistance to fluconazole. Microbiology 146(Pt 11), 2743-2754.

Cannon, R. D., Lamping, E., Holmes, A. R., Niimi, K., Baret, P. V., Keniya, M. V., et al. (2009). Efflux-mediated antifungal drug resistance. Clin. Microbiol. Rev. 22, 291-321. doi: 10.1128/CMR.00051-08

Chaudry, A. N., Travers, P. J., Yuenger, J., Colletta, L., Evans, P., Zenilman, J. M., et al. (2004). Analysis of vaginal acetic acid in patients undergoing treatment for bacterial vaginosis. J. Clin. Microbiol. 42, 5170-5175. doi: 10.1128/JCM.42.11.5170-5175.2004

Chen, K. H., Miyazaki, T., Tsai, H. F., and Bennett, J. E. (2007). The bZip transcription factor Cgaplp is involved in multidrug resistance and required for activation of multidrug transporter gene CgFLR1 in Candida glabrata. Gene 386, 63-72. doi: 10.1016/j.gene.2006.08.010

Costa, C., Henriques, A., Pires, C., Nunes, J., Ohno, M., Chibana, H., et al. (2013a). The dual role of Candida glabrata drug:H+ antiporter CgAqrl (ORF CAGLOJ09944g) in antifungal drug and acetic acid resistance. Front. Microbiol. 4:170. doi: 10.3389/fmicb.2013.00170

Costa, C., Nunes, J., Henriques, A., Mira, N. P., Nakayama, H., Chibana, H., et al. (2014). Candida glabrata drug:H+ antiporter CgTpo3 (ORF CAGLOI10384g): role in azole drug resistance and polyamine homeostasis. J. Antimicrob. Chemother. doi: 10.1093/jac/dku1044. [Epub ahead of print].

Costa, C., Pires, C., Cabrito, T. R., Renaudin, A., Ohno, M., Chibana, H., et al. (2013b). Candida glabrata Drug:H+ Antiporter CgQdr2 confers imidazole drug resistance, being activated by transcription factor CgPdr1. Antimicrob. Agents Chemother. 57, 3159-3167. doi: 10.1128/AAC.00811-12

Dias, P. J., and Sá-Correia, I. (2013). The drug: $\mathrm{H}(+)$ antiporters of family 2 (DHA2), siderophore transporters (ARN) and glutathione: $\mathrm{H}(+)$ antiporters (GEX) have a common evolutionary origin in hemiascomycete yeasts. BMC Genomics 14:901. doi: 10.1186/1471-2164-14-901

Dias, P. J., and Sá-Correia, I. (in press). Phylogenetic and syntenic analyses of the 12-spanner drug:H+ antiporter family 1 (DHA1) in pathogenic Candida species: evolution of MDR1 and FLU1 genes. Genomics. (accepted).

Dias, P. J., Seret, M. L., Goffeau, A., Sá-Correia, I., and Baret, P. V. (2010). Evolution of the 12-spanner drug:H+ antiporter DHAl family in hemiascomycetous yeasts. OMICS 14, 701-710. doi: 10.1089/omi.2010.0104
Ernst, R., Kueppers, P., Stindt, J., Kuchler, K., and Schmitt, L. (2010). Multidrug efflux pumps: substrate selection in ATP-binding cassette multidrug efflux pumps-first come, first served? FEBS J. 277, 540-549. doi: 10.1111/j.17424658.2009.07485.x

Felder, T., Bogengruber, E., Tenreiro, S., Ellinger, A., Sá-Correia, I., and Briza, P. (2002). Dtrlp, a multidrug resistance transporter of the major facilitator superfamily, plays an essential role in spore wall maturation in Saccharomyces cerevisiae. Eukaryot. Cell 1, 799-810. doi: 10.1128/EC.1.5.799-810.2002

Gautam, P., Shankar, J., Madan, T., Sirdeshmukh, R., Sundaram, C. S., Gade, W. N., et al. (2008). Proteomic and transcriptomic analysis of Aspergillus fumigatus on exposure to amphotericin B. Antimicrob. Agents Chemother. 52, 4220-4227. doi: 10.1128/AAC.01431-07

Goffeau, A., Barrell, B. G., Bussey, H., Davis, R. W., Dujon, B., Feldmann, H., et al. (1996). Life with 6000 genes. Science 274, 546, 563-547. doi: 10.1126/science.274.5287.546

Goldway, M., Teff, D., Schmidt, R., Oppenheim, A. B., and Koltin, Y. (1995). Multidrug resistance in Candida albicans: disruption of the $B E N r$ gene. Antimicrob. Agents Chemother. 39, 422-426. doi: 10.1128/AAC.39.2.422

Gupta, V., Kohli, A., Krishnamurthy, S., Puri, N., Aalamgeer, S. A., Panwar, S., et al. (1998). Identification of polymorphic mutant alleles of CaMDR1, a major facilitator of Candida albicans which confers multidrug resistance, and its in vitro transcriptional activation. Curr. Genet. 34, 192-199. doi: $10.1007 / \mathrm{s} 002940050385$

Hayashi, K., Schoonbeek, H. J., and De Waard, M. A. (2002). Bcmfs1, a novel major facilitator superfamily transporter from Botrytis cinerea, provides tolerance towards the natural toxic compounds camptothecin and cercosporin and towards fungicides. Appl. Environ. Microbiol. 68, 4996-5004. doi: 10.1128/AEM.68.10.4996-5004.2002

Hiller, D., Sanglard, D., and Morschhauser, J. (2006). Overexpression of the MDR1 gene is sufficient to confer increased resistance to toxic compounds in Candida albicans. Antimicrob. Agents Chemother. 50, 1365-1371. doi: 10.1128/AAC.50.4.1365-1371.2006

Karababa, M., Coste, A. T., Rognon, B., Bille, J., and Sanglard, D. (2004). Comparison of gene expression profiles of Candida albicans azole-resistant clinical isolates and laboratory strains exposed to drugs inducing multidrug transporters. Antimicrob. Agents Chemother. 48, 3064-3079. doi: 10.1128/AAC.48.8.3064-3079.2004

Li, R., Kumar, R., Tati, S., Puri, S., and Edgerton, M. (2013). Candida albicans flul-mediated efflux of salivary histatin 5 reduces its cytosolic concentration and fungicidal activity. Antimicrob. Agents Chemother. 57, 1832-1839. doi: 10.1128/AAC.02295-12

Liu, T. T., Znaidi, S., Barker, K. S., Xu, L., Homayouni, R., Saidane, S., et al. (2007). Genome-wide expression and location analyses of the Candida albicans Taclp regulon. Eukaryot. Cell 6, 2122-2138. doi: 10.1128/EC.00327-07

Markovich, S., Yekutiel, A., Shalit, I., Shadkchan, Y., and Osherov, N. (2004). Genomic approach to identification of mutations affecting caspofungin susceptibility in Saccharomyces cerevisiae. Antimicrob. Agents Chemother. 48, 3871-3876. doi: 10.1128/AAC.48.10.3871-3876.2004

Morschhauser, J. (2010). Regulation of multidrug resistance in pathogenic fungi. Fungal Genet. Biol. 47, 94-106. doi: 10.1016/j.fgb.2009.08.002

Nunes, P. A., Tenreiro, S., and Sá-Correia, I. (2001). Resistance and adaptation to quinidine in Saccharomyces cerevisiae: role of QDR1 (YIL120w), encoding a plasma membrane transporter of the major facilitator superfamily required for multidrug resistance. Antimicrob. Agents Chemother. 45, 1528-1534. doi: 10.1128/AAC.45.5.1528-1534.2001

Roohparvar, R., De Waard, M. A., Kema, G. H., and Zwiers, L. H. (2007). MgMfs1, a major facilitator superfamily transporter from the fungal wheat pathogen Mycosphaerella graminicola, is a strong protectant against natural toxic compounds and fungicides. Fungal Genet. Biol. 44, 378-388. doi: 10.1016/j.fgb.2006.09.007

Sá-Correia, I., Santos, S. C., Teixeira, M. C., Cabrito, T. R., and Mira, N. M. (2009). Drug: $\mathrm{H}^{+}$antiporters in chemical stress response in yeast. Trends Microbiol. 17, 22-31. doi: 10.1016/j.tim.2008.09.007

Sanglard, D., Ischer, F., Monod, M., and Bille, J. (1997). Cloning of Candida albicans genes conferring resistance to azole antifungal agents: characterization of CDR2, a new multidrug ABC transporter gene. Microbiology 143 (Pt 2), 405-416. doi: 10.1099/00221287-143-2-405

Sanglard, D., Kuchler, K., Ischer, F., Pagani, J. L., Monod, M., and Bille, J. (1995). Mechanisms of resistance to azole antifungal agents in Candida albicans isolates 
from AIDS patients involve specific multidrug transporters. Antimicrob. Agents Chemother. 39, 2378-2386. doi: 10.1128/AAC.39.11.2378

Shah, A. H., Singh, A., Dhamgaye, S., Chauhan, N., Vandeputte, P., Suneetha, K. J., et al. (2014). Novel role of a family of major facilitator transporters in biofilm development and virulence of Candida albicans. Biochem. J. 460, 223-235. doi: 10.1042/BJ20140010

Teixeira, M. C., Cabrito, T. R., Hanif, Z. M., Vargas, R. C., Tenreiro, S., and SáCorreia, I. (2011). Yeast response and tolerance to polyamine toxicity involving the drug: $\mathrm{H}^{+}$antiporter Qdr3 and the transcription factors Yap1 and Gcn4. Microbiology 157, 945-956. doi: 10.1099/mic.0.043661-0

Teixeira, M. C., Dias, P. J., Monteiro, P. T., Sala, A., Oliveira, A. L., Freitas, A. T., et al. (2010). Refining current knowledge on the yeast FLR1 regulatory network by combined experimental and computational approaches. Mol. Biosyst. 6, 2471-2481. doi: 10.1039/c004881j

Teixeira, M. C., Dias, P. J., Simoes, T., and Sá-Correia, I. (2008). Yeast adaptation to mancozeb involves the up-regulation of FLR1 under the coordinate control of Yap1, Rpn4, Pdr3, and Yrr1. Biochem. Biophys. Res. Commun. 367, 249-255. doi: 10.1016/j.bbrc.2007.12.056

Tenreiro, S., Nunes, P. A., Viegas, C. A., Neves, M. S., Teixeira, M. C., Cabral, M. G., et al. (2002). AQR1 gene (ORF YNL065w) encodes a plasma membrane transporter of the major facilitator superfamily that confers resistance to shortchain monocarboxylic acids and quinidine in Saccharomyces cerevisiae. Biochem. Biophys. Res. Commun. 292, 741-748. doi: 10.1006/bbrc.2002.6703

Tenreiro, S., Rosa, P. C., Viegas, C. A., and Sá-Correia, I. (2000). Expression of the AZR1 gene (ORF YGR224w), encoding a plasma membrane transporter of the major facilitator superfamily, is required for adaptation to acetic acid and resistance to azoles in Saccharomyces cerevisiae. Yeast 16, 1469-1481. doi: 10.1002/1097-0061(200012)16:16\&lt;1469::AID-YEA640\&gt;3.0.CO;2-A

Tomitori, H., Kashiwagi, K., Asakawa, T., Kakinuma, Y., Michael, A. J., and Igarashi, K. (2001). Multiple polyamine transport systems on the vacuolar membrane in yeast. Biochem. J. 353, 681-688. doi: 10.1042/0264-6021:3530681

Tyms, A. S. (1989). "Polyamines and the growth of bacteria and viruses," in The Physiology of Polyamines, eds U. Bachrach and Y. M. Heimer (Boca Raton, FL: CRC Press), 3-33.

Vargas, R. C., Garcia-Salcedo, R., Tenreiro, S., Teixeira, M. C., Fernandes, A. R., Ramos, J., et al. (2007). Saccharomyces cerevisiae multidrug resistance transporter Qdr2 is implicated in potassium uptake, providing a physiological advantage to quinidine-stressed cells. Eukaryot. Cell 6, 134-142. doi: 10.1128/EC.00290-06
Velasco, I., Tenreiro, S., Calderon, I. L., and Andre, B. (2004). Saccharomyces cerevisiae Aqr1 is an internal-membrane transporter involved in excretion of amino acids. Eukaryot. Cell 3, 1492-1503. doi: 10.1128/EC.3.6.1492-15 03.2004

Wirsching, S., Michel, S., Kohler, G., and Morschhauser, J. (2000a). Activation of the multiple drug resistance gene MDR1 in fluconazole-resistant, clinical Candida albicans strains is caused by mutations in a trans-regulatory factor. J. Bacteriol. 182, 400-404. doi: 10.1128/JB.182.2.400-404.2000

Wirsching, S., Michel, S., and Morschhauser, J. (2000b). Targeted gene disruption in Candida albicans wild-type strains: the role of the MDR1 gene in fluconazole resistance of clinical Candida albicans isolates. Mol. Microbiol. 36, 856-865. doi: 10.1046/j.1365-2958.2000.01899.x

Wirsching, S., Moran, G. P., Sullivan, D. J., Coleman, D. C., and Morschhauser, J. (2001). MDR1-mediated drug resistance in Candida dubliniensis. Antimicrob. Agents Chemother. 45, 3416-3421. doi: 10.1128/AAC.45.12.34163421.2001

Yamada-Okabe, T., and Yamada-Okabe, H. (2002). Characterization of the CaNAG3, CaNAG4, and CaNAG6 genes of the pathogenic fungus Candida albicans: possible involvement of these genes in the susceptibilities of cytotoxic agents. FEMS Microbiol. Lett. 212, 15-21. doi: 10.1111/j.15746968.2002.tb11238.x

Conflict of Interest Statement: The authors declare that the research was conducted in the absence of any commercial or financial relationships that could be construed as a potential conflict of interest.

Received: 26 March 2014; accepted: 09 May 2014; published online: 28 May 2014. Citation: Costa C, Dias PJ, Sá-Correia I and Teixeira MC (2014) MFS multidrug transporters in pathogenic fungi: do they have real clinical impact? Front. Physiol. 5:197. doi: 10.3389/fphys.2014.00197

This article was submitted to Membrane Physiology and Membrane Biophysics, a section of the journal Frontiers in Physiology.

Copyright () 2014 Costa, Dias, Sá-Correia and Teixeira. This is an open-access article distributed under the terms of the Creative Commons Attribution License (CC BY). The use, distribution or reproduction in other forums is permitted, provided the original author(s) or licensor are credited and that the original publication in this journal is cited, in accordance with accepted academic practice. No use, distribution or reproduction is permitted which does not comply with these terms. 